\title{
BMJ Open Recorded atypical hallucinations in psychotic and affective disorders and associations with non-benzodiazepine hypnotic use: the South London and Maudsley Case Register
}

Karen Isabel Birnie, ${ }^{1}$ Robert Stewart, ${ }^{2,3}$ Anna Kolliakou ${ }^{2}$

To cite: Birnie KI, Stewart $\mathrm{R}$, Kolliakou A. Recorded atypical hallucinations in psychotic and affective disorders and associations with nonbenzodiazepine hypnotic use: the South London and Maudsley Case Register. BMJ Open 2018;8:e025216. doi:10.1136/ bmjopen-2018-025216

- Prepublication history for this paper is available online. To view these files, please visit the journal online (http://dx.doi. org/10.1136/bmjopen-2018025216).

Received 8 July 2018 Revised 20 August 2018 Accepted 23 August 2018

Check for updates

(C) Author(s) (or their employer(s)) 2018. Re-use permitted under CC BY-NC. No commercial re-use. See rights and permissions. Published by BMJ.

${ }^{1}$ Department of Health Psychology, King's College London, London, UK ${ }^{2}$ Department of Psychological Medicine, King's College London, London, UK ${ }^{3}$ South London and Maudsley NHS Foundation Trust, London, UK

Correspondence to Karen Isabel Birnie; karen.birnie@kcl.ac.uk

\section{ABSTRACT}

Objectives Hallucinations are present in many conditions, notably psychosis. Although under-researched, atypical hallucinations, such as tactile, olfactory and gustatory (TOGHs), may arise secondary to hypnotic drug use, particularly non-benzodiazepine hypnotics ('Z drugs'). This retrospective case-control study investigated the frequency of TOGHs and their associations with prior Z drug use in a large mental healthcare database.

Methods TOGHs were ascertained in 2014 using a bespoke natural language processing algorithm and were analysed against covariates (including use of $Z$ drugs, demographic factors, diagnosis, disorder severity and other psychotropic medications) ascertained prior to 2014. Results In 43339 patients with International Classification of Diseases, Tenth Edition schizophreniform or affective disorder diagnoses, $324(0.75 \%)$ had any TOGH recorded ( $0.54 \%$ tactile, $0.24 \%$ olfactory, $0.06 \%$ gustatory hallucinations). TOGHs were associated with male gender, black ethnicity, schizophreniform diagnosis and higher disorder severity on Health of the National Outcome Scales. In fully adjusted models, tactile and olfactory hallucinations remained independently associated with prior mention of $Z$ drugs (ORs 1.86 and 1.60, respectively). Conclusions We successfully developed a natural language processing algorithm to identify instances of TOGHs in the clinical record. TOGHs overall, tactile and olfactory hallucinations were shown to be associated with prior mention of $Z$ drugs. This may have implications for the diagnosis and treatment of patients with comorbid sleep and psychiatric conditions.

\section{INTRODUCTION}

Although hallucinations are not necessarily abnormal experiences, they are commonly associated with psychotic and organic disorders. ${ }^{2}$ They can occur in any sensory modality, although the most commonly reported hallucinations in patients with schizophrenia are auditory and visual with estimated prevalences of around $70 \%$, and ranging from $15 \%$ to $60 \%$, respectively. ${ }^{3} 4$ Similar prevalences have been described in
Strengths and limitations of this study

- This was a large study involving 43339 patients.

- The prospective study design reduced recall bias.

- The natural language processing (NLP) tool, developed to recognise references to tactile, olfactory and gustatory hallucinations (TOGHs) from a naturalistic data source, has great potential for applicability in other data sources.

- This study used routine healthcare records, thus requiring clinicians to recognise and record TOGHs accurately.

- We were not able to conclude that medication use always antedated TOGHs.

bipolar disorder (approximately $70 \%$ and $25 \%$, respectively). ${ }^{5}$ Tactile, olfactory and gustatory hallucinations (TOGHs) are recognised to occur in psychotic disorders, but have received substantially less investigation. In samples with either schizophrenia or severe mental illness more generally, tactile hallucination prevalences have ranged from $15 \%$ to $27 \%,{ }^{36-8}$ and are recognised to be associated with substance abuse and withdrawal regardless of diagnosis. ${ }^{9}$ The prevalence of olfactory hallucinations has ranged from $15 \%$ to $27 \%,{ }^{6} 7$ although this is likely to be an underestimate because of the tendency for traditional questionnaires to examine hallucinations generally rather than by modality. ${ }^{10}$ The prevalence of gustatory hallucinations has ranged from $4 \%$ to $14 \% .{ }^{6}$ Hypnotic use in the general population has been found to be associated with higher reported tactile and gustatory hallucinations ${ }^{11}$ and hallucinations in all/most modalities have been described as increased in people receiving non-benzodiazepine hypnotics, specifically ' $\mathrm{Z}$ drugs' (zolpidem, zopiclone or zaleplon), ${ }^{12} 13$ possibly potentiated by other psychotropic agents. ${ }^{14}$ 
However, these observations have largely been derived case studies and small samples.

The aim of this study was to ascertain the frequency of TOGHs in a large sample of people with schizophreniform or affective disorder diagnoses receiving mental health services, and to test a hypothesised association between TOGHs and $\mathrm{Z}$ drug use.

\section{METHODS}

\section{Setting}

The South London and Maudsley NHS Foundation Trust (SLaM) is one of Europe's largest mental healthcare providers, serving a geographic catchment of four South London boroughs (Croydon, Lambeth, Lewisham and Southwark) with approximately 1.2 million residents. Since 2006, an electronic health records system has been used throughout SLaM, and the Clinical Record Interactive Search (CRIS) system, developed in 2008, allows researchers to retrieve de-identified information from these records for around 280000 cases to date. ${ }^{15} 16$ CRIS has been approved as a database for secondary analysis by the Oxfordshire Research Ethics Committee C (Ethics ID: 08/H0606/71+5) and a service user-led committee provides oversight for projects using these data. ${ }^{17}$

\section{Study population}

The baseline sample derived from CRIS comprised a cohort of all patients, aged 18-65 years on 1 January 2014, who had received a diagnosis of a schizophrenia-related disorder (extracted using ICD-10 code: F2x) and/or a mood/affective disorder (ICD-10 code: F3x) prior to that date. Case records from the cohort were then searched for instances of TOGHs between 1 January 2014 and 31 December 2014.

\section{Patient involvement}

We did not directly incorporate patient and public involvement (PPI) into this particular study but the SLaM BRC Case Register used in the analysis was developed with extensive PPI and is overseen by a committee that includes service-user representatives.

\section{Outcome: tactile, olfactory and gustatory hallucinations}

A pre-existing natural language processing algorithm designed to detect mentions of hallucinations in text fields ${ }^{18}$ was further adapted to search and retrieve references to TOGHs. Three keywords were added to search for references to hallucinations mentioned in the freetext fields of CRIS (including written assessments, progress notes and correspondence). These were 'olfactory', 'tactile' or 'gustat*' (and 'hallucinations'). The application query retrieved 20924 instances across CRIS; 1000 randomly selected instances were annotated for reference to TOGHs, of which 300 instances were double-annotated resulting in a kappa statistic of 0.83 (95\% CI 0.71 to 0.89 ). The information extraction algorithm was developed using 500 annotated records (training set), seeking to identify linguistic patterns indicating a true reference to any of the three hallucination types (ie, rather than negation statements, irrelevant mentions or mentions of the symptom occurring in someone other than the patient). Having developed the algorithm using General Architecture for Text Engineering (GATE) software, ${ }^{19}$ it was tested on another 500 annotated records (gold standard set), and achieved a precision score (positive predictive value) of 0.91 and a recall (sensitivity) score of 1.00. Deploying the algorithm over the complete dataset of 20924 instances, 17066 were identified as true positive references to TOGHs. Binary outcome variables were thereby created representing the occurrence of hallucinations in each or any modality (olfactory, tactile, gustatory, any of the three).

\section{Covariates}

Covariates were extracted to indicate status at the index date (1 January 2014). Demographic factors comprised age, gender and recorded ethnicity-the latter categorised into three groups: black background (including Caribbean, African and any other black background), white background (British, Irish and any other white background) and other (including mixed or multiple ethnic backgrounds).

Regarding clinical factors, the sample was categorised into those who had previously received a diagnosis of schizophreniform disorder, a mood disorder diagnosis or both. In addition, we made use of the Health of the Nations Outcome Scales (HoNOS) assessment, routinely administered in UK mental health services, ${ }^{20}$ including the total score (an indicator of overall functioning), and the score relating to alcohol and substance use problems (categorising the problem as present on the basis of a score of 2 or more on the $0-4$ scale), extracting scores closest to the index date.

Hypnotic agent use before the index date was determined from structured medication fields in the record, supplemented by a natural language processing algorithm which ascertains recorded pharmacotherapy from open-text fields. Ascertained agents were classified into $\mathrm{Z}$ drugs (zopiclone, zaleplon and zolpidem) and all other hypnotics licensed for use in insomnia or where sedation is needed (diazepam, flurazepam, loprazolam, lorazepam, lormetazepam, nitrazepam, temazepam, trimipramine, doxepin, chloral hydrate, clomethiazole, melatonin, promethazine hydrochloride). Antidepressant use during the same period was identically extracted, and classified into two groups: those acting primarily through the serotonergic system (citalopram, escitalopram, paroxetine, fluoxetine, fluvoxamine, sertraline, duloxetine, venlafaxine, trazodone, amitriptyline, clomipramine, dosulepin, doxepin, imipramine, trimipramine, mianserin, mirtazapine, isocarboxazid, phenelzine, tranylcypromine, moclobemide, and agomelatin) and those with other primary targets (non-serotonergicreboxetine, flupentixol and nortriptyline). ${ }^{21}$ 


\section{Analyses}

Percentages were used to describe all categorical variables. Mean and SD were used to describe age and HoNOS total scores were described by median and IQR; $\chi^{2}$ test statistics were calculated to identify any differences in sociodemographic and clinical characteristics between patients with or without TOGHs overall and for each modality. Binomial logistic regression models were used to test the association between overall/individual TOGHs and mention of $\mathrm{Z}$ drugs. Sociodemographic and clinical characteristics associated with individual outcomes at $\mathrm{p}<0.05$ were entered in the final regression model. Because of missing data pertaining to HoNOS scores, a post hoc analysis was conducted, restricting the sample to those with scores present before investigating the impact of adjusting for HoNOS-derived covariates. All analyses were conducted using Stata V.13. ${ }^{22}$

\section{RESULTS}

The analysed sample consisted of 43339 patients. The mean age was 41.4 years (SD 12.2) and $53.5 \%$ of the sample was female. One-quarter of the sample was of a black background and $57.9 \%$ were of a white background. Regarding previous diagnosis, over two-thirds had received a mood disorder diagnosis only $(67.0 \%)$ and $24.4 \%$ a schizophreniform diagnosis only; $22.6 \%$ of the total sample had mention of $\mathrm{Z}$ drugs in their record and $25.7 \%$ had mention of other hypnotic drugs. The median of the HoNOS total scores was 8 (IQR 0-40).

The natural language processing algorithm identified 324 patients in the sample with at least one modality of TOGH recorded in 2014 case notes: $0.75 \%$ of the sample. Within this group, tactile hallucinations were most common $(71.6 \%)$, followed by olfactory $(31.8 \%)$ and gustatory $(7.4 \%)$ modalities. The proportion of patients who reported any TOGH did not differ significantly from the remainder of the sample by age $\left(\chi^{2}(3)=5.22\right.$, $\mathrm{p}=0.157)$ or HoNOS problems with drugs and alcohol $\left(\chi^{2}(1)=2.78, \mathrm{p}=0.095\right)$ but did with respect to gender $\left(\chi^{2}(1)=5.66, \mathrm{p}=0.017\right)$ and ethnicity $\left(\chi^{2} \quad(2)=74.30\right.$, $\mathrm{p}<0.001)$, with over-representation of men and patients from black ethnic groups (table 1). In regard to clinical features, significant differences were observed between patients with any TOGH and the rest of the sample with respect to diagnosis $\left(\chi^{2}(2)=272.97, \mathrm{p}<0.001\right)$, and were more likely to have recorded $\mathrm{Z}$ drug use $\left(\chi^{2}(1)=118.78\right.$, $\mathrm{p}<0.001)$, other hypnotic use $\left(\chi^{2}(1)=149.54, \mathrm{p}<0.001\right)$ and non-serotonergic antidepressant use $\left(\chi^{2}(1)=23.81\right.$, $\mathrm{p}<0.001)$. Prevalence of any TOGH by diagnostic group was $1.5 \%$ in $\mathrm{F} 2,0.24 \%$ in $\mathrm{F} 3 \%$ and $1.7 \%$ in those with both diagnoses recorded.

In unadjusted regression analysis (table 2), recorded $\mathrm{Z}$ drug use was significantly associated with any TOGH (OR 3.17, 95\% CI 2.54 to 3.95), tactile (OR 3.22, 95\% CI 2.49 to 4.17 ), olfactory (OR $2.77,95 \%$ CI 1.88 to 4.09 ) and gustatory hallucinations (OR 3.43, 95\% CI 1.54 to 7.63) specifically. After adjusting for confounders, all four models remained significant, although attenuated, with recorded $\mathrm{Z}$ drugs associated with any TOGH mention (OR 2.02, 95\% CI 1.57 to 2.77), tactile (OR 2.09, 95\% CI 1.57 to 2.78 ), olfactory (OR $1.69,95 \%$ CI 1.10 to 2.59 ) and gustatory hallucinations (OR 2.58, 95\% CI 1.06 to $6.28)$.

Restricting the subsample to patients who had a HoNOS total score available in the clinical record $(n=26201)$, after adjusting for the confounders indicated in table 2, associations with recorded $\mathrm{Z}$ drug use remained significant for any TOGH (OR 1.82, 95\% CI 1.41 to 2.34), tactile (OR 1.81, 95\% CI 1.41 to 2.34) and olfactory hallucinations (OR 1.60, 95\% CI 1.01 to 2.54) but not for gustatory hallucinations (OR 2.19, 95\% CI 0.87 to 5.55). Further adjustment for HoNOS total scores and HoNOS problems with drugs and alcohol in this regression model did not alter findings substantially for any TOGH (OR 1.80, 95\% CI 1.40 to 2.32 ), tactile (OR $1.86,95 \%$ CI 1.38 to 2.51 ), olfactory (OR 1.60, 95\% CI 1.01 to 2.54) or gustatory hallucinations (OR 2.19, 95\% CI 0.86 to 5.55) (table 3).

\section{DISCUSSION}

In a large database derived from mental healthcare electronic records, we investigated the 1-year period frequency of recorded tactile, olfactory and gustatory hallucinations in patients with schizophreniform or affective disorders, having developed an algorithm to detect recorded mentions of these symptoms with a reasonable level of accuracy. Because of the relative rarity of these symptoms, large administrative databases present an opportunity for better definition and aetiological investigation. However, symptom profiles are not routinely recorded in structured fields within mental health records, so the development and application of natural language processing techniques are similarly essential for improving understanding-rendering information available at scale which would have previously been unrealised, and generating databases which are both large and detailed. ${ }^{18} 23$

Frequencies within the sample were $0.54 \%, 0.24 \%$ and $0.06 \%$ for tactile, olfactory and gustatory hallucinations, respectively. These are clearly substantially lower than prevalence reports of $15 \%-27 \%$ for tactile hallucinations in samples with severe mental illness, ${ }^{367} 15 \%-19 \%$ for olfactory hallucinations in schizophrenia ${ }^{6} 8$ and $4 \%-9 \%$ for gustatory hallucinations. ${ }^{68}$ However, research to date has ascertained these symptoms from lifetime recollections derived from fully structured diagnostic questionnaires, using specific questions relating to these hallucinations, ${ }^{3} 67$ whereas our estimates were 1 year rates of reference to TOGHs, derived from information contained in routine healthcare records. It should be noted that clinical records likely underestimate the prevalence of TOGHs, given that they may not be recorded systematically in clinical practice. The relatively broadly defined sample should also be borne in mind, as, consistent with other reports, the frequencies of TOGHs were substantially higher in patients who had received 
Table 1 Demographic and clinical characteristics of total and individual samples

\begin{tabular}{|c|c|c|c|c|c|}
\hline & \multirow[b]{2}{*}{$\begin{array}{l}\text { Total sample } \\
(n=43339)\end{array}$} & \multicolumn{4}{|c|}{ Groups with TOGH } \\
\hline & & $\begin{array}{l}\text { Any TOGH } \\
(n=324)\end{array}$ & $\begin{array}{l}\text { Tactile } \\
\text { (n=232) }\end{array}$ & $\begin{array}{l}\text { Olfactory } \\
(n=103)\end{array}$ & $\begin{array}{l}\text { Gustatory } \\
\text { (n=24) }\end{array}$ \\
\hline \multicolumn{6}{|l|}{ Age (years), \% } \\
\hline $18-31$ & 24.8 & 29.0 & 28.4 & 35.9 & 20.8 \\
\hline $32-41$ & 25.4 & 27.2 & 29.7 & 19.4 & 29.2 \\
\hline $42-50$ & 24.7 & 22.5 & 21.1 & 26.2 & 16.7 \\
\hline $51-65$ & 25.1 & 21.3 & 20.7 & 18.4 & 33.3 \\
\hline Female gender, \% & 53.5 & 46.9 & 41.8 & 54.4 & 62.5 \\
\hline \multicolumn{6}{|l|}{ Ethnicity, \% } \\
\hline Black & 25.0 & 45.1 & 46.1 & 46.6 & 37.5 \\
\hline White & 57.9 & 38.6 & 37.1 & 37.9 & 41.7 \\
\hline Other & 17.1 & 16.0 & 16.4 & 15.5 & 20.8 \\
\hline \multicolumn{6}{|l|}{ Diagnosis, \% } \\
\hline F2 & 24.3 & 54.9 & 56.0 & 55.3 & 50.0 \\
\hline F3 & 67.0 & 24.1 & 26.3 & 17.5 & 20.8 \\
\hline Both & 8.6 & 21.0 & 17.7 & 27.2 & 29.2 \\
\hline \multicolumn{6}{|l|}{ Medication use, \% } \\
\hline$Z$ drugs & 22.6 & 47.8 & 48.3 & 44.7 & 50.0 \\
\hline Other hypnotics & 25.7 & 55.2 & 56.0 & 54.4 & 45.8 \\
\hline Serotonin-related antidepressants & 54.6 & 54.3 & 55.2 & 50.5 & 54.2 \\
\hline Non-serotonin antidepressants & 4.2 & 9.6 & 10.3 & 10.7 & 0 \\
\hline \multicolumn{6}{|l|}{ HoNOS } \\
\hline Total (median (IQR) & $8.0(0-40)^{\star}$ & $10(0-34) \dagger$ & $10(0-34) \ddagger$ & $9(0-29) \S$ & $6(0-24)$ ๆ \\
\hline Problem with alcohol and drug use \% & $15.2^{\star \star}$ & $18.6+\dagger$ & 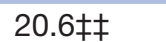 & $15.1 \S \S$ & $13.69 \emptyset$ \\
\hline
\end{tabular}

${ }^{*} \mathrm{n}=26201$.

$\dagger \mathrm{t}=284$.

$\ddagger \mathrm{n}=204$.

$\S \mathrm{n}=86$.

丹n $=21$.

${ }^{*} \mathrm{n}=27047$.

$\dagger \dagger n=295$.

$\ddagger \ddagger n=209$.

$\S \S n=93$.

१ๆ $\mathrm{n}=22$.

HoNOS, Health of the Nations Outcome Scales; TOGH, tactile, olfactory or gustatory hallucinations.

a schizophreniform diagnosis alone or in combination ( $1.5 \%$ and $1.7 \%$ prevalences, respectively), compared with that in patients who had received an affective disorder diagnosis alone $(0.24 \%){ }^{6}$

We also specifically investigated associations with $\mathrm{Z}$ drug (zopiclone, zolpidem, zaleplon) hypnotic use, given previous cited associations with hallucinations. Neuropsychiatric adverse effects of $\mathrm{Z}$ drugs, including hallucinations and psychosis, have been described for over 15 years, ${ }^{24-27}$ including atypical and/or multimodal hallucinations. ${ }^{28}$ Most often this has been related to zolpidem, although this may reflect higher usage rates. ${ }^{29}$ While mechanisms underlying other adverse effects, such as parasomnias have been investigated ${ }^{30}$ the explanation for associations with hallucinations remains unclear, although it does not appear to be related directly to dose or plasma concentrations, and at least some excess adverse event reporting has been suggested in association with media exposure. ${ }^{31}$ Interactions have also been reported with other psychotropic agents. ${ }^{32}$ In line with our hypothesis, patients with use of $\mathrm{Z}$ drugs mentioned in their previous clinical record were more likely to experience TOGHs overall and tactile and olfactory hallucinations specifically. Associations with gustatory hallucinations appeared similar in strength but were not statistically significant in all models because of the relative rarity of hallucinations in this modality being recorded. Unlike previous studies, which have tended to involve patient interviews subsequent to drug use, our analysis investigated associations between mentions of drug categories at any time preceding 2014 and recorded 
Table 2 Adjusted logistic regression model assessing the association between Z drug use and TOGH

\begin{tabular}{|c|c|c|c|c|c|c|c|c|}
\hline & \multicolumn{2}{|l|}{ All modalities } & \multicolumn{2}{|l|}{ Tactile } & \multicolumn{2}{|l|}{ Olfactory } & \multicolumn{2}{|l|}{ Gustatory } \\
\hline & OR (95\% Cl) & $P$ values & OR $(95 \% \mathrm{Cl})$ & P values & OR $(95 \% \mathrm{CI})$ & P values & OR (95\% Cl) & $P$ values \\
\hline$Z$ drugs & $\begin{array}{l}2.02 \\
\text { (1.59 to } 2.57)\end{array}$ & $<0.001$ & $\begin{array}{l}2.09 \\
\text { (1.57 to } 2.77 \text { ) }\end{array}$ & $<0.001$ & $\begin{array}{l}1.69 \\
\text { (1.10 to } 2.59)\end{array}$ & 0.016 & $\begin{array}{l}2.58 \\
\text { (1.06 to } 6.28)\end{array}$ & 0.036 \\
\hline Gender & $\begin{array}{l}0.90 \\
(0.71 \text { to } 1.12)\end{array}$ & 0.347 & $\begin{array}{l}1.12 \\
(0.86 \text { to } 1.47)\end{array}$ & 0.402 & - & - & - & - \\
\hline \multicolumn{9}{|l|}{ Age (years) } \\
\hline $18-31$ & - & - & - & - & $\begin{array}{l}2.11 \\
\text { (1.22 to } 3.64)\end{array}$ & 0.008 & - & - \\
\hline $42-51$ & - & - & - & - & $\begin{array}{l}1.31 \\
\text { (0.73 to } 2.35)\end{array}$ & 0.355 & - & - \\
\hline $51-65$ & - & - & - & - & $\begin{array}{l}0.99 \\
(0.52 \text { to } 1.85)\end{array}$ & 0.963 & - & - \\
\hline Black & $\begin{array}{l}1.66 \\
\text { (1.30 to } 2.13)\end{array}$ & $<0.001$ & $\begin{array}{l}1.82 \\
\text { (1.36 to } 2.45)\end{array}$ & $<0.001$ & $\begin{array}{l}1.57 \\
\text { (1.02 to } 2.43)\end{array}$ & 0.042 & - & - \\
\hline Other & $\begin{array}{l}1.34 \\
(0.97 \text { to } 1.86)\end{array}$ & 0.080 & $\begin{array}{l}1.45 \\
(0.98 \text { to } 2.12)\end{array}$ & 0.060 & $\begin{array}{l}1.23 \\
(0.68 \text { to } 2.21)\end{array}$ & 0.492 & - & - \\
\hline \multicolumn{9}{|l|}{ Diagnosis } \\
\hline F3 & Ref & & Ref & & Ref & & Ref & \\
\hline $\mathrm{F} 2$ & $\begin{array}{l}4.82 \\
\text { (3.61 to } 6.45)\end{array}$ & $<0.001$ & $\begin{array}{l}4.04 \\
(2.90 \text { to } 5.64)\end{array}$ & $<0.001$ & $\begin{array}{l}6.63 \\
\text { (3.81 to } 11.55)\end{array}$ & $<0.001$ & $\begin{array}{l}6.30 \\
(2.19 \text { to } 18.14)\end{array}$ & 0.001 \\
\hline F2/F3 & $\begin{array}{l}3.86 \\
\text { ( } 2.73 \text { to } 5.47 \text { ) }\end{array}$ & $<0.001$ & $\begin{array}{l}2.72 \\
(1.79 \text { to } 4.16)\end{array}$ & $<0.001$ & $\begin{array}{l}7.44 \\
(3.97 \text { to } 13.95)\end{array}$ & $<0.001$ & $\begin{array}{l}8.21 \\
(2.47 \text { to } 27.28)\end{array}$ & 0.001 \\
\hline
\end{tabular}

En dash denoted variable not entered in regression model.

$\mathrm{TOGH}$, tactile, olfactory or gustatory hallucinations.

TOGHs during 2014. A large multinational general population survey found that overall hypnotic use was associated with tactile and gustatory hallucinations; however, we only found independent evidence of other hypnotics (non-Z drugs) being associated with tactile hallucinations (table 2).

Secondary findings suggest that other factors, such as diagnosis and ethnicity, may play a role in susceptibility to TOGHs. These associations have not been identified in previous research, although this may reflect differences in sample size, since higher non-Caucasian ethnicity was found in one previous study, ${ }^{6}$ and lower levels of TOGHs were found in patients from India compared with the USA in another study, ${ }^{7}$ which may suggest some international/ethnic variation in prevalence. In final models (table 3), higher HoNOS total score was associated with TOGHs generally, which has some concordance with findings from other studies of associations with more severe psychotic syndromes (particularly in relation to delusional symptoms), ${ }^{6}$ although this was only present for tactile hallucinations.
Strengths of the study include the large sample size, the prospective study design, the range of covariates assessed and the naturalistic source of data. The development of the natural language processing algorithm was successful, resulting in a tool that can be used to automatically extract data on TOGHs from the electronic health record, in addition to the growing number of other information extraction algorithms being developed. ${ }^{16} 18$ Given the relative simplicity of the underlying construct (TOGHs) being ascertained from text fields, we would anticipate good cross-applicability to other data sources. The sample itself ought also to be reasonably generalisable, coming from a mixed inner and outer urban catchment area with high social diversity. ${ }^{16}$

The nature of the source data needs to be borne in mind when drawing conclusions; in particular, the fact that hallucinations were ascertained from routine mental healthcare records rather than from a research instrument. Presence of TOGHs in the CRIS database therefore depends on a clinician asking about or noticing the symptom, on their recording it, and on their recording 
Table 3 Adjusted logistic regression model assessing the association between Z drug use and TOGH in the subsample of patients with HoNOS data present

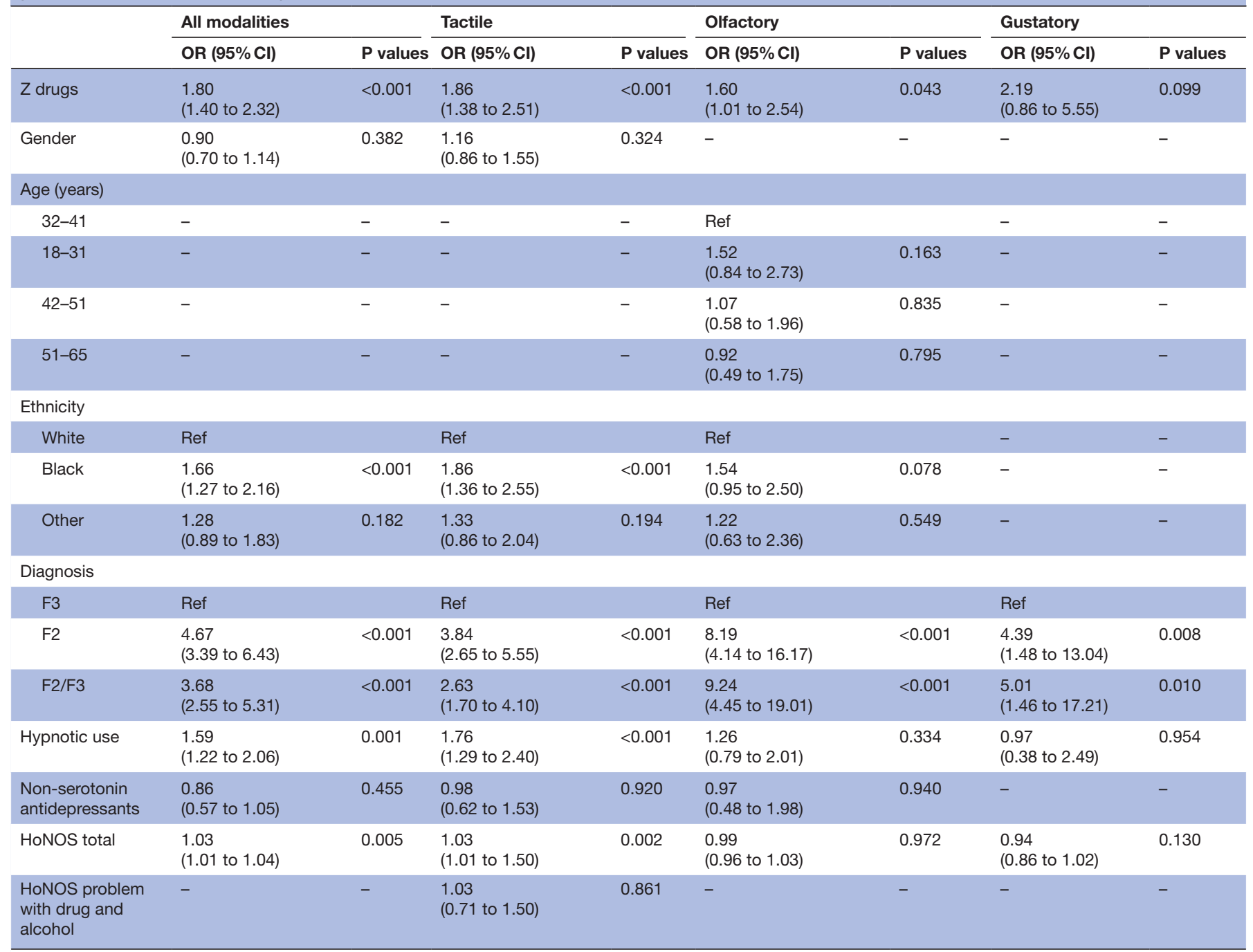

En dash denoted variable not entered in regression model.

HoNOS, Health of the Nations Outcome Scales; TOGH, tactile, olfactory or gustatory hallucinations.

it in such a way that it was successfully extracted by the natural language processing algorithm (ie, recorded as a phenomenological term: eg, 'tactile hallucination' rather than 'feels that insects are crawling over his skin'). As described, the algorithm itself was efficient at identifying these terms, with high precision and recall statistics; however, symptoms will have been missed if they went unnoticed or unrecorded in clinical care. The alternative approach in this field has been to evaluate them in a recruited sample with a formal questionnaire, and to our knowledge, this is how all previous prevalence estimates have been derived, most often using the Structured Clinical Interview for DSM. ${ }^{36}$ However, this approach generally depends on responses to one or two questions in a long interview schedule administered for other purposes and deriving lifetime rather than current occurrences. There is therefore considerable potential for recall bias, as well as potential selection bias arising from the sample recruited which are less likely to influence data from routine healthcare. Ultimately, it has not yet been established whether data extracted from clinical records are an underestimate of true prevalence, or whether recalled self-report data elicited from diagnostic instruments are an overestimate, and this requires further evaluation.

Considering the hypothesis under investigation, while the prospective study design reduces effects of recall bias, whereby participants may have had difficulty remembering details about their hallucinations or medication use, establishing causality is difficult. Although mentions of medication were ascertained prior to the observation period, we were not able to conclude that medication use always antedated hallucinations. In addition, medication, while mentioned, was not necessarily used, and dosage was not accounted for; neither were different $\mathrm{Z}$ drugs distinguished. In addition, patients prescribed certain drugs may have more clinical contact and care around prescribing, perhaps leading to decreased vulnerability. 
This study could be improved in the future by fine-tuning of the application, for example, including other keywords that may indicate the presence of TOGHs-terms such as disturbance or unusual experience/sensation, may identify more instances of TOGHs. The development of an application that explored medication use, not just mentions, could be used to determine the nature of any relationship with hallucinations. Further research could also explore differences between genders, given the sex-related pharmacokinetics of zolpidem. ${ }^{33}$ This would be valuable information for patients who are known to be at risk of unusual sensory phenomena, where clinicians could make more informed decisions about prescribing. Our findings are preliminary but if replicated could have implications for patients with comorbid disordered sleep or mood symptoms.

Acknowledgements The NIHR Biomedical Research Centre at the South London and Maudsley NHS Foundation Trust and King's College London provided core support in the development and delivery of the study.

Contributors This research was completed as part of an intercalated BSc in Psychology. KIB, AK and RS all contributed to the study design and manuscript preparation. KB took primary responsibility for data collection and analysis. All authors contributed to and have approved the final manuscript.

Funding RS and AK are part-funded by the National Institute for Health Research (NIHR) Biomedical Research Centre at South London and Maudsley NHS Foundation Trust and King's College London.

Competing interests None declared.

Patient consent Not required.

Provenance and peer review Not commissioned; externally peer reviewed.

Data sharing statement There are no additional unpublished data available.

Open access This is an open access article distributed in accordance with the Creative Commons Attribution Non Commercial (CC BY-NC 4.0) license, which permits others to distribute, remix, adapt, build upon this work non-commercially, and license their derivative works on different terms, provided the original work is properly cited, appropriate credit is given, any changes made indicated, and the use is non-commercial. See: http://creativecommons.org/licenses/by-nc/4.0/.

\section{REFERENCES}

1. Barnes J, David AS. Visual hallucinations in Parkinson's disease: a review and phenomenological survey. J Neurol Neurosurg Psychiatry 2001;70:727-33.

2. Bassiony MM, Steinberg MS, Warren A, et al. Delusions and hallucinations in Alzheimer's disease: prevalence and clinical correlates. Int J Geriatr Psychiatry 2000;15:99-107.

3. Mueser KT, Bellack AS, Brady EU. Hallucinations in schizophrenia. Acta Psychiatr Scand 1990;82:26-9.

4. Bracha HS, Wolkowitz OM, Lohr JB, et al. High prevalence of visual hallucinations in research subjects with chronic schizophrenia. Am J Psychiatry 1989;146:526-8.

5. Baethge C, Baldessarini RJ, Freudenthal K, et al. Hallucinations in bipolar disorder: characteristics and comparison to unipolar depression and schizophrenia. Bipolar Disord 2005;7:136-45.

6. Lewandowski KE, DePaola J, Camsari GB, et al. Tactile, olfactory, and gustatory hallucinations in psychotic disorders: a descriptive study. Ann Acad Med Singapore 2009;38:383-5.

7. Thomas P, Mathur P, Gottesman II, et al. Correlates of hallucinations in schizophrenia: A cross-cultural evaluation. Schizophr Res 2007(92(1-3):41-9.
8. Chaudhury S. Hallucinations: Clinical aspects and management. Ind Psychiatry J 2010;19:5-12.

9. Brewer JD, Meves A, Bostwick JM, et al. Cocaine abuse: dermatologic manifestations and therapeutic approaches. J Am Acad Dermatol 2008;59:483-7.

10. Arguedas D, Langdon R, Stevenson R. Neuropsychological characteristics associated with olfactory hallucinations in schizophrenia. J Int Neuropsychol Soc 2012;18:799-808.

11. Ohayon MM. Prevalence of hallucinations and their pathological associations in the general population. Psychiatry Res 2000/97(23):153-64.

12. Olsen LG, Olson L. Hypnotic hazards: adverse effects of zolpidem and other z-drugs. Aust Prescr 2008;31:146-9.

13. Dart RC. Medical toxicology: Lippincott Williams \& Wilkins, 2004.

14. Elko CJ, Burgess JL, Robertson WO. Zolpidem-associated hallucinations and serotonin reuptake inhibition: a possible interaction. J Toxicol Clin Toxicol 1998;36:195-203.

15. Stewart R, Soremekun M, Perera G, et al. The South London and Maudsley NHS Foundation Trust Biomedical Research Centre (SLAM BRC) case register: development and descriptive data. BMC Psychiatry 2009;9:1-12

16. Perera G, Broadbent M, Callard F, et al. Cohort profile of the South London and Maudsley NHS Foundation Trust Biomedical Research Centre (SLaM BRC) Case Register: current status and recent enhancement of an Electronic Mental Health Record-derived data resource. BMJ Open 2016:6:e008721.

17. Fernandes AC, Cloete D, Broadbent MT, et al. Development and evaluation of a de-identification procedure for a case register sourced from mental health electronic records. BMC Med Inform Decis Mak 2013:13:71.

18. Jackson RG, Patel R, Jayatilleke $N$, et al. Natural language processing to extract symptoms of severe mental illness from clinica text: the Clinical Record Interactive Search Comprehensive Data Extraction (CRIS-CODE) project. BMJ Open 2017;7:e012012.

19. Cunningham H, Tablan V, Roberts A, et al. Getting more out of biomedical documents with GATE's full lifecycle open source text analytics. PLoS Comput Biol 2013:9:e1002854.

20. Wing JK, Lelliott P, Beevor AS. Progress on HoNOS. British Journal of Psychiatry 2000:176:392-3.

21. BMJ Group. British National Formulatory (BNF), 2014;67.

22. Statacorp. Stata Statistical Software: Release. College Station, TX: StataCorp LP, 2013:13;13.

23. Stewart R. The big case register. Acta Psychiatr Scand 2014;130:83-6.

24. Duggal HS. New-onset transient hallucinations possibly due to eszopiclone: a case study. Prim Care Companion J Clin Psychiatry 2007;9:468-9.

25. Stone JR, Zorick TS, Tsuang J. Dose-related illusions and hallucinations with zaleplon. Clin Toxicol 2008:46:344-5.

26. Tsai MJ, Huang YB, Wu PC. A novel clinical pattern of visual hallucination after zolpidem use. J Toxicol Clin Toxicol 2003;41:869-72.

27. Ben-Hamou M, Marshall NS, Grunstein RR, et al. Spontaneous adverse event reports associated with zolpidem in Australia 20012008. J Sleep Res 2011;20:559-68.

28. Ram D, Eiman N, Gowdappa B. Multimodal Hallucination (Audiovisual, Kinaesthetic and Scenic) Associated with the Use of Zolpidem. Clin Psychopharmacol Neurosci 2015;13:215-7.

29. Dolder CR, Nelson MH. Hypnosedative-Induced Complex Behaviours. CNS Drugs 2008;22:1021-36.

30. Hoque R, Chesson AL. Zolpidem-induced sleepwalking, sleep related eating disorder, and sleep-driving: fluorine-18flourodeoxyglucose positron emission tomography analysis, and a literature review of other unexpected clinical effects of zolpidem. $J$ Clin Sleep Med 2009;5:471-6.

31. Wong CK, Marshall NS, Grunstein RR, et al. Spontaneous Adverse Event Reports Associated with Zolpidem in the United States 2003-2012. JCSM 2016;13:223-34.

32. Gunja N. The clinical and forensic toxicology of Z-drugs. J Med Toxicol 2013:9:155-62.

33. Norman JL, Fixen DR, Saseen JJ, et al. Zolpidem prescribing practices before and after Food and Drug Administration required product labeling changes. SAGE Open Med 2017;5:205031211770768. 